\title{
Duração do aleitamento materno exclusivo associado a anemia por deficiência de ferro em crianças: uma revisão sistemática
}

\author{
Duration of exclusive breastfeeding associated with iron deficiency anemia in children: a \\ systematic review
}

Duración de la lactancia materna exclusiva asociada con anemia por deficiencia de hierro en niños: una revisión sistemática

leda Aparecida Diniz ${ }^{1 *}$, Kelly de Freitas Santos ${ }^{1}$, Nayara Ragi Baldoni ${ }^{1}$, Eduardo José Braga ${ }^{1}$, Carolina Aparecida Alves Bueno ${ }^{1}$, Andressa Castanheira Barcelos ${ }^{2}$, Nathália Ribeiro Lima ${ }^{3}$, Daniela Aparecida de Faria1, Márcia Christina Caetano Romano1, Joel Alves Lamounier ${ }^{1}$.

\section{RESUMO}

Objetivo: Identificar por meio de uma revisão sistemática a associação da duração do aleitamento materno exclusivo com a anemia ferropriva. Métodos: Trata-se de uma revisão sistemática da literatura realizada de acordo com a diretriz de Itens de Relatório Preferenciais para Revisões Sistemáticas e Meta-análises (PRISMA). As estratégias de buscas foram realizadas a partir da estrutura PECO. Utilizou-se o software Rayyan para leitura de título e resumo dos artigos, e o escore de qualidade metodológico proposto por Donws \& Black na leitura na íntegra. Os dados foram analisados de forma descritiva. Resultados: Identificou-se 3.039 artigos, e ao final, considerou-se um total de 28 manuscritos publicados entre 1978 e 2020. $60,7 \%$ dos estudos apresentaram associação estatística entre a duração do aleitamento materno exclusivo e anemia por deficiência de ferro. $46,4 \%$ relataram introdução complementar aos seis meses de idade, $25 \%$ revelaram introdução precoce de alimentos e $28,6 \%$ não avaliaram alimentação complementar. Considerações finais: A maioria dos estudos analisados apontaram associação estatisticamente significativa entre a duração do aleitamento materno exclusivo até os seis meses de idade e anemia ferropriva. Contudo, esses achados devem ser analisados com cautela, considerando que variáveis que podem interferir no desfecho da anemia por deficiência de ferro foram pouco exploradas.

Palavras-chave: Anemia, Aleitamento materno exclusivo, Lactente.

\begin{abstract}
Objective: To identify, through a systematic review, the association between the duration of exclusive breastfeeding and iron deficiency anemia. Methods: This is a systematic literature review performed in accordance with the Preferred Reporting Items for Systematic Reviews and Meta-analyses (PRISMA) guideline. The search strategies were carried out from the PECO structure. The Rayyan software was used to read the titles and abstracts of the articles, and the methodological quality score proposed by Donws \& Black was used in the full reading. Data were analyzed descriptively. Results: 3,039 articles were identified, and in the end, a total of 28 manuscripts published between 1978 and 2020 were considered. $60.7 \%$ of the studies showed a statistical association between the duration of exclusive breastfeeding and iron deficiency anemia. $46.4 \%$ reported complementary introduction at six months of age, $25 \%$ reported early introduction of foods
\end{abstract}

\footnotetext{
1 Universidade Federal de São João Del Rei, Divinópolis - MG *E-mail: ieda.diniz@yahoo.com.br

${ }^{2}$ Complexo de Saúde São João de Deus, Divinópolis - MG.

${ }^{3}$ Hospital e Maternidade Santa Mônica, Divinópolis - MG.
}

SUBMETIDO EM: 1/2022

PUBLICADO EM: 2/2022 
and $28.6 \%$ did not evaluate complementary feeding. Final considerations: Most of the studies analyzed showed a statistically significant association between the duration of exclusive breastfeeding up to six months of age and iron deficiency anemia. However, these findings should be analyzed with caution, considering that variables that may interfere with the outcome of iron deficiency anemia have been little explored.

Keywords: Anemia, Exclusive breastfeeding, Infant.

\section{RESUMEN}

Objetivo: Identificar, a través de una revisión sistemática, la asociación entre la duración de la lactancia materna exclusiva y la anemia ferropénica. Métodos: Esta es una revisión sistemática de la literatura realizada de acuerdo con la guía Preferred Reporting Items for Systematic Reviews and Meta-analyses (PRISMA). Las estrategias de búsqueda se realizaron a partir de la estructura PECO. Se utilizó el software Rayyan para la lectura de los títulos y resúmenes de los artículos, y en la lectura completa se utilizó el puntaje de calidad metodológica propuesto por Donws \& Black. Los datos se analizaron descriptivamente. Resultados: se identificaron 3.039 artículos y finalmente se consideraron un total de 28 manuscritos publicados entre 1978 y 2020. El 60,7\% de los estudios mostró asociación estadística entre la duración de la lactancia materna exclusiva y la anemia ferropénica. El 46,4\% informó introducción complementaria a los seis meses de edad, el $25 \%$ informó introducción temprana de alimentos y el $28,6 \%$ no evaluó alimentación complementaria. Consideraciones finales: La mayoría de los estudios analizados mostraron una asociación estadísticamente significativa entre la duración de la lactancia materna exclusiva hasta los seis meses de edad y la anemia ferropénica. Sin embargo, estos hallazgos deben ser analizados con cautela, considerando que las variables que pueden interferir en el desenlace de la anemia ferropénica han sido poco exploradas.

Palabras clave: Anemia, Lactancia materna exclusiva, Lactante.

\section{INTRODUÇÃO}

A anemia, segundo a World Health Organization (WHO) (2015), se define por valores de hemoglobina $(\mathrm{Hb})$ sérica abaixo do esperado para idade, gênero, estado fisiológico e altitude e pode ocorrer por diversos fatores, como a deficiência de ferro e de outros micronutrientes, processos patológicos e infecciosos, e uso de medicamentos que prejudiquem a absorção do ferro (WHO, 2017). Embora seja de causa multifatorial, a deficiência de ferro tem sido considerada um dos principais problemas nutricionais de relevância à saúde pública associado a anemia, tanto de países desenvolvidos como aqueles em desenvolvimento (CAI C, et al., 2017).

A prevalência global de anemia em crianças de seis a 59 meses é de $42,6 \%$. As estimativas deste relatório sugerem que a anemia afeta cerca de 800 milhões de crianças e mulheres (WHO, 2015). No Brasil estimase que a prevalência de anemia entre crianças de até 83,9 meses é de $33 \%$. Em relação às regiões brasileiras a prevalência de anemia apresenta-se menor na região Sudeste (28\%), e se assemelha nas regiões Norte / Centro-Oeste (36\%), Nordeste (38\%) e Sul (35\%) (NOGUEIRA-DE-ALMEIDA CA, et al., 2021).

O ferro apresenta funções indispensáveis como transporte de oxigênio e elétrons para as células, além de integrar os sistemas enzimáticos de diversos tecidos. Ademais, sua deficiência pode prejudicar o desenvolvimento infantil, as habilidades cognitivas e comportamentais, linguagem e capacidade motora, e a resposta imune. Esses danos podem permanecer mesmo após o tratamento do agravo, por curto, médio ou longo prazo, podendo até mesmo ser irreversíveis (FISBERG M, et al., 2018).

A Sociedade Brasileira de Pediatria (SBP) (2018) e a WHO (2017) ressaltam que durante os primeiros dois anos de vida, a criança apresenta rápido crescimento da estrutura física, o que demanda um aporte elevado de ferro, e explica a anemia ferropriva afetar especialmente as crianças de seis a 24 meses, exigindo estratégias efetivas de prevenção e combate voltadas às necessidades desse público (CASTRO IRR, et al., 2021).

Recentes estudos têm investigado as múltiplas causas predisponentes à anemia que incluem aspectos socioeconômicos e ambientais, anemia na gestação, além de outros fatores biológicos e nutricionais (SALAMI 
A, et al., 2018; ANDRE HP, et al., 2018). Segundo as Diretrizes Brasileiras sobre anemia ferropriva, o incentivo a alimentação adequada, ao aleitamento materno exclusivo até os seis meses e complementado até os dois anos ou mais, como também a restrição de uso de leite de vaca antes dos doze meses de idade são condições protetoras à anemia (FISBERG M, et al., 2018).

Apesar de poucos dados disponíveis na literatura nacional, estudos internacionais indicam dados controversos e afirmam que o aleitamento materno exclusivo até os seis meses de idade ou mais aumenta o risco de anemia por deficiência de ferro. Deste modo os beneficios do aleitamento materno em manter o nível de $\mathrm{Hb}$ seria sugerido por estes estudos até os quatro meses de idade (DOMELLOF M, et al., 2014; CHEN, CM, et al., 2020).

Agregando-se a esses achados, a Sociedade Brasileira de Pediatria recomenda a suplementação de ferro com $1 \mathrm{mg} / \mathrm{kg} /$ dia a partir de três meses para os lactentes que apresentarem alguma condição de risco, e aos seis meses de idade para aqueles que não apresentam (SBP, 2021). Em contrapartida, outras pesquisas não corroboram com essa suposição e, de fato, mostram resultados contrários, evidenciando que o aleitamento materno exclusivo por mais tempo está associado a um melhor status de ferro nas crianças de até 24 meses de idade (PÉREZ-ESCAMILLA R, et al., 2019; UYOGA MA, et al., 2016).

Diante do exposto, uma investigação sobre o tema pode contribuir para o conhecimento e desvelamento da questão, aprimorando a literatura sobre o tema, além de trazer indicadores para prevenção de agravos decorrentes da duração do aleitamento materno exclusivo na anemia ferropriva. O estudo teve como objetivo identificar, na literatura, a associação da duração do aleitamento materno exclusivo com a anemia ferropriva e a deficiência de ferro.

\section{MÉTODOS}

Trata-se uma revisão sistemática da literatura realizada de acordo com a diretriz de Itens de Relatório Preferenciais para Revisões Sistemáticas e Meta-análises (PRISMA) (PAGE MJ, et al., 2021). A investigação foi registrada no International Prospect Register of Systematic Reviews (PROSPERO), registo CRD42020186422.

Como critérios de elegibilidade, a pesquisa buscou responder à pergunta: $\mathrm{A}$ duração do aleitamento materno exclusivo influencia a ocorrência de anemia ferropriva em crianças de até 36 meses de idade?. A partir da pergunta norteadora foi construída a estrutura PECO (Pessoas: lactentes, Exposição: aleitamento materno exclusivo, Comparação: outros tipos de aleitamento, Resultados/Outcomes: anemia). Os critérios de inclusão foram estudos observacionais com data de publicação até 17 de agosto de 2020. Não houve limitação temporal nessa revisão por considerar que estudos com relevância podem ter sido publicados em tempos distintos.

Foram considerados elegíveis os estudos sobre duração do aleitamento materno exclusivo associado ao nível de hemoglobina entre lactentes. Não houve restrição de idioma. Estudos de revisões integrativas, revisões sistemáticas, relatórios, editoriais, estudos qualitativos, estudos experimentais, livros, relatórios, teses, dissertações, monografias, resumos publicados em Anais congressos/simpósio, estudos cujos participantes eram crianças nascidas prematuras ou com relato de anemias que não fossem causadas por deficiência de ferro foram excluídos.

As estratégias de buscas foram criadas a partir do PECO, sendo as palavras-chaves contidas no Medical Subject Headings (MeSH). Os MeSH e entretermos foram combinados com os operadores booleanos AND e OR (Quadro 1 e 2 Suplementar). Nas bases de dados, US National Library of Medicine National Institutes of Health (PubMed), Scopus, Science Direct, Web of Science e Biblioteca Virtual de Saúde (BVS), a busca foi realizada na opção "Busca avançada".

Após aprovação do protocolo de pesquisa no PROSPERO, realizou-se de forma independente as buscas das publicações. Os manuscritos foram exportados na mesma data da busca para o software Rayyan (OUZZANI M, et al., 2016). Após a exportação, as publicações duplicadas foram excluídas, e a seleção dos artigos foi realizada pela leitura de título e resumo de forma independente. No caso de discordância entre os pesquisadores, um terceiro pesquisador avaliou o manuscrito e em consenso decidiu-se pela inclusão ou exclusão do estudo na revisão. 
Posterior a seleção por títulos e resumos os estudos foram lidos na íntegra para extração de dados para análise. As variáveis coletadas foram: autor, ano de publicação, tipo de estudo, tamanho da amostra, duração do aleitamento materno exclusivo, ponte de corte do valor da hemoglobina para considerar anemia, ponto de corte do valor da ferritina para considerar deficiência de ferro, descrição do uso de suplementação de ferro, e do início da alimentação complementar.

$\mathrm{Na}$ fase de leitura na íntegra os artigos foram avaliados em relação a qualidade metodológica. Este processo foi realizado pelo escore de qualidade metodológico proposto por Downs SH e Black N (1998), o instrumento avalia a validade interna, validade externa e poder estatístico do estudo por meio de 27 itens de pontuação. Ao considerar que a revisão em questão avalia estudos observacionais, cinco itens que dizem respeito somente a estudos experimentais foram retirados. Dessa forma 22 itens foram avaliados, e considerou-se para esta revisão os estudos que apresentaram classificação acima de 70\%, o que equivale a 16 pontos no escore (BALDONI NR, et al., 2019). A avaliação da qualidade foi realizada por dois pesquisadores de forma independente e contou com um terceiro pesquisador para decisão de inclusão ou exclusão do artigo no caso de divergência da avaliação da qualidade entre os pesquisadores.

Para a avaliaçao do nível de evidência dos manuscritos considerou-se a preconização da Agency for Health Care Research and Quality (AHRQ). Este instrumento estabelece a força de evidência de acordo com o desenho de estudo. Cabe ressaltar que o maior nível de evidência considerado pelo AHRQ são os estudos de metanálise, e os com menor força de evidência referem-se aqueles geradas por opiniões de especialistas. Conforme sugere o instrumento estudos de coorte e seccionais, como no caso desta revisão, recebem uma pontuação de 3 e 4 pontos respectivamente (AHRQ, 2008; GALVÃO CM, 2006)

Após a leitura, os dados foram extraídos de cada estudo e inseridos em planilha do Microsoft Excel. O desfecho de interesse foi anemia por deficiência de ferro associado a duração do aleitamento materno exclusivo. Os dados foram analisados de forma descritiva.

Cabe ressaltar que esta revisão foi atualizada em novembro de 2021 com o propósito de inclusão de manuscritos inéditos publicados a partir de agosto de 2020. Estes estudos foram incluídos na seção de discussão desta revisão após leitura e certificação da qualidade metodológica. Nesta atualização foram identificados três artigos que respondiam aos critérios de inclusão e apresentavam potencial para contribuir com a presente revisão.

\section{RESULTADOS}

Ao todo foram identificados 3.039 artigos correspondentes às cinco bases de dados: Pubmed, Science Direct, Web of Science, Scopus e BVS (Tabela 1).

Tabela 1 - Número de publicações identificadas em cada base de dados.

\begin{tabular}{lcc}
\hline Base de dados & Número & $\%$ \\
\hline Science Direct & 171 & 5,6 \\
Web of Science & 497 & 16,4 \\
Scopus & 1.663 & 54,7 \\
Pubmed nova versão & 442 & 14,5 \\
Portal BVS* & 266 & 8,7 \\
\hline Total & $\mathbf{3 . 0 3 9}$ & $\mathbf{1 0 0}$
\end{tabular}

Legenda: * Portal BVS - 266 (MEDLINE - 232/ LILACS - 21/ WHO IRIS - 5/ IBECS - 4/ BINACS - 1/CUMED -1/ PAHO - 1/ PAHO IRIS - 1). Fonte: Diniz IA, et al., 2022.

Deste total foram retiradas 692 publicações em duplicatas. Após a leitura de resumo e título foram excluídos 2.304 estudos que não atendiam aos critérios de inclusão. Para análise foram considerados 44 manuscritos elegíveis para a leitura na íntegra. Destes, foram identificados cinco estudos experimentais e um artigo não relacionado com o objetivo proposto. Outro artigo não foi possível acesso ao texto, foram realizadas tentativas de contato com os autores para recuperação do manuscrito via e-mail, contudo, sem êxito. Do total de 37 artigos, nove foram excluídos pela qualidade metodológica inferior a $70 \%$. Conforme ilustrado no fluxograma foram considerados um total de 28 artigos (Figura 1). 
Figura 1 - Fluxograma das fases da Revisão Sistemática.

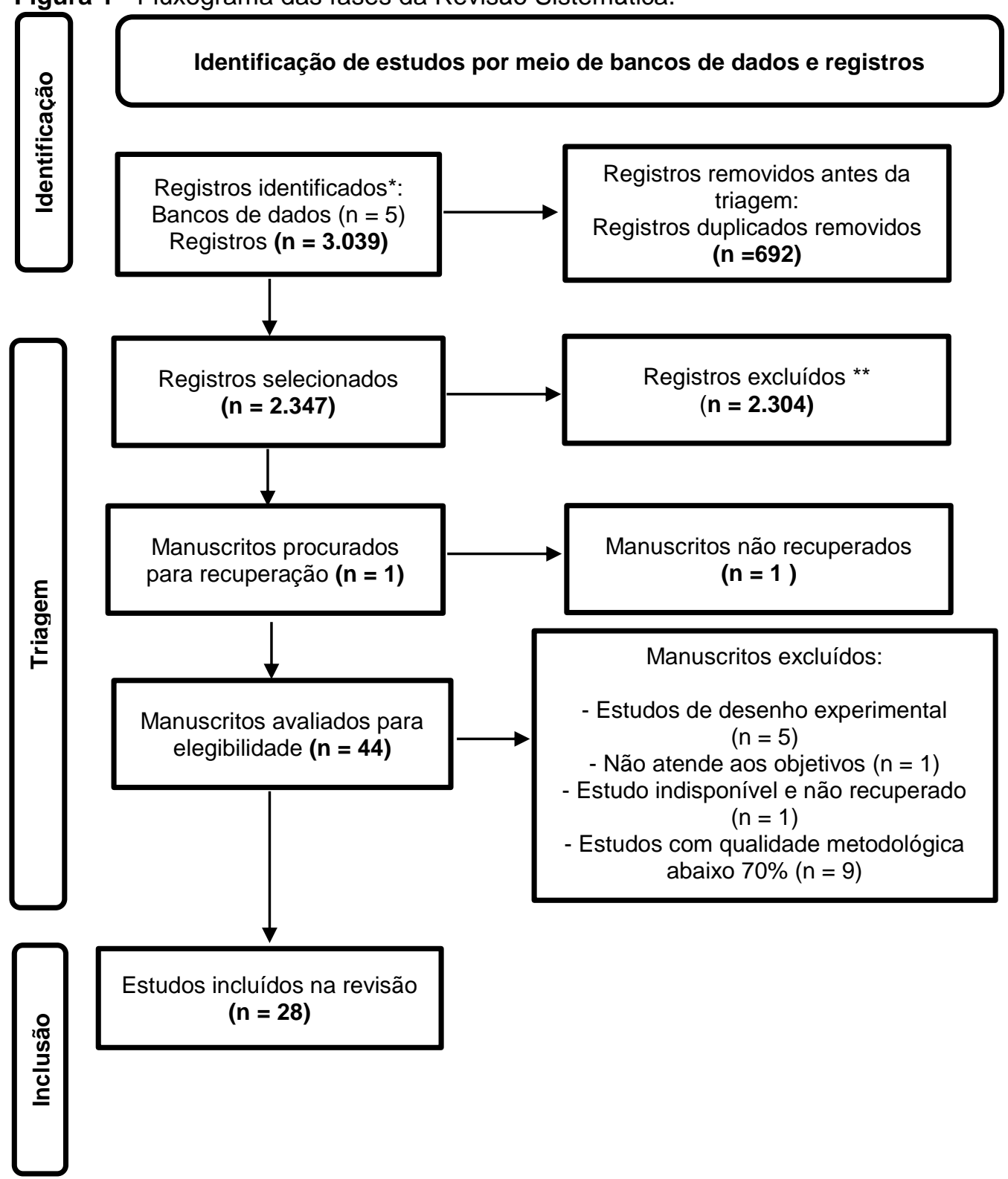

Fonte: Diniz IA, et al., 2022; baseado no guideline PRISMA de Page MJ, et al., 2021.

Os artigos elegíveis incluídos na revisão foram publicados entre 1978 e 2020. Em relação a temporalidade, não houve publicações na década de 1980 e nos anos 2018 e 2019 . Pode-se observar um discreto aumento de publicações no ano de 2011 comparados aos outros anos, n=3 (10,7\%) (CAPOZZI L, et al., 2011; ISOMURA H, et al., 2011; POLAT TB, et al., 2011).

Somados, os estudos analisados foram em sua maioria realizados em países em desevolvimento $\mathrm{n}=12$ $(42,8 \%)$ e subdesenvolvidos $n=5$ (17,8\%) (ANTUNES $H$, et al., 2002; ASSIS AMO, et al., 2004; MONTERROSA EC, et al., 2008; GONG YH, et al., 2008; LUO R, et al., 2014; JIA N, et al., 2015; DALILI H, et al., 2015; WANG F, et al., 2016; CLARK KM, et al., 2017; HONG J, et al., 2017; WANG J, et al., 2020; CHEN CM, et al.,2020, TANTRACHEEWATHORN S e LOHAJAROENSUB S, 2005; SITI-NOOR AS, et al., 2006; BÜLBÜL I, et al., 2008; POLATTB, et al., 2011; UYOGA MA, et al., 2016).

O país que apresentou maior número de publicações foi a China com sete manuscritos (25\%) (GONG YH, et al., 2008; LUO R, et al., 2014; JIA N, et al., 2015; WANG F, et al., 2016; CLARK KM, et al., 2017.; WANG J, et al., 2020; CHEN CM, et al., 2020). Em sequência o Japão apresentou três artigos (10,7\%) (HOKAMA T, 1993; ISOMURA H, et al., 2011; KIMURA M, et al., 2018). Do total de estudos treze apresentaram delineamento transversal (46, 4\%) (ANTUNES H, et al., 2002; ASSIS AMO, et al., 2004; CHRISTOFIDES A, 
et al., 2005; SITI-NOOR AS, et al., 2006; CHANTRY CJ, et al., 2007; BÜLBÜL I, et al., 2008; POLAT TB, et al., 2011; LUO R, et al., 2014; JIA N, et al., 2015; DALILI H, et al., 2015; UYOGA MA, et al., 2016; HONG J, et al., 2017; CHEN CM, et al., 2020), treze (46, 4\%) eram coortes prospectivas (SAARINEN UM, 1978; HOKAMA T, 1993; WANDEL M, et al., 1996; THORSDOTTIR I, et al., 2003; TANTRACHEEWATHORN S e LOHAJAROENSUB S, 2005; HOPKINS D, et al., 2007; MONTERROSA EC, et al., 2008; GONG YH, et al., 2008; CAPOZZI L, et al., 2011; ISOMURA H, et al., 2011; GONDOLF UH, et al., 2013; WANG F, et al., 2016; CLARK KM, et al., 2017). Dois eram estudo de caso-controle (7,1\%) (WANG J, et al., 2020; KIMURA M, et al., 2018). Os estudos apresentaram nível de evidência três ou quatro.

A população investigada nos estudos apresentou uma amostra que, somada, incluíram 78.753 crianças avaliadas. Os participantes se encontram na faixa etária entre zero e 36 meses. Os estudos de coorte apresentaram baseline ao nascimento e acompanhamentos que diferiram entre doze e 24 meses. Entre os estudos de desenho transversal as idades dos participantes oscilaram entre seis e 36 meses, e entre os estudos de caso-controle de zero a 24 meses (Quadro 1). 
Quadro 1 - Descrição dos artigos analisados.

\begin{tabular}{|c|c|c|c|c|}
\hline Referência/ ano de publicação & País & $\begin{array}{l}\text { Desenho do } \\
\text { estudo }\end{array}$ & Descrição da Amostra & $\begin{array}{c}\text { Qualidade } \\
\text { metodológica e } \\
\text { nível de evidência }\end{array}$ \\
\hline Saarinen U, 1978 & Finlândia & Coorte Prospectiva & $\begin{array}{c}238 \text { crianças nascidos entre } 38 \text { e } 42 \text { semanas e peso ao nascer } \\
\text { superior a } 3,0 \mathrm{~kg}\end{array}$ & 17 pontos/ Nível 3 \\
\hline Hokama T, 1993 & Japão & Coorte Prospectiva & 356 bebês saudáveis nascidos a termo, peso ao nascer $>2500 \mathrm{~g}$. & 16 pontos/ Nível 3 \\
\hline WandeL M, et al, 1996 & Noruega & Coorte Prospectiva & $\mathbf{7 4}$ bebês ( 35 noruegueses e 39 de famílias de imigrantes) & 16 pontos/ Nível 3 \\
\hline Antunes $\mathrm{H}$, et al., 2002 & Brasil & Transversal & 188 lactentes com nove meses de idade & 16 pontos/ Nível 4 \\
\hline Thorsdottir I, et al., 2003 & Islândia & Coorte Prospectiva & 171 crianças de zero a 12 meses de idade & 18 pontos/ Nível 3 \\
\hline Assis AMO, et al., 2004 & Brasil & Transversal & 553 crianças & 19 pontos/ Nível 4 \\
\hline Christofides A, et al., 2005 & Canadá & Transversal & 170 crianças de quatro a 18 meses de idade & 17 pontos/ Nível 4 \\
\hline $\begin{array}{l}\text { Tantracheewathorn S e } \\
\text { Lohajaroensub S, } 2005\end{array}$ & Tailândia & Coorte Prospectiva & 40 bebês nascidos a termo & 17 pontos/ Nível 3 \\
\hline Siti-Noor AS, et al., 2006 & Malásia & Transversal & 490 lactentes de oito a 26 meses & 20 pontos/ Nível 4 \\
\hline Chantry CJ, et al., 2007 & Estados Unidos & Transversal & 2.268 crianças de seis a 24 meses & 19 pontos/ Nível 4 \\
\hline Hopkins D, et al., 2007 & Inglaterra & Coorte Prospectiva & 1.710 crianças (928 de 8 meses e, 782 de 12 meses). & 19 pontos/ Nível 3 \\
\hline Monterrosa EC, et al., 2008 & México & Coorte prospectiva & 146 pares elegíveis de mãe e filho. & 19 pontos/ Nível 3 \\
\hline Gong YH, et al., 2008 & China & Coorte prospectiva & 1.001 recém-nascidos normais e saudáveis & 17 pontos/ Nível 3 \\
\hline Bülbül I, et al., 2008 & Turquia & Transversal & 650 crianças sem intercorrências ao nascimento & 19 pontos/ Nível 4 \\
\hline Capozzi L, et al., 2011 & Itália & Coorte prospectiva & 121 crianças saudáveis & 16 pontos/ Nível 3 \\
\hline Isomura H, et al., 2011 & Japão & Coorte prospectiva & 103 recém-nascidos & 16 pontos/ Nível 3 \\
\hline Polat TB, et al., 2011 & Turquia & Transversal & 611 bebês & 17 pontos/ Nível 4 \\
\hline Gondolf UH, et al., 2013 & Dinamarca & Coorte prospectiva & 312 bebês & 18 pontos/ Nível 3 \\
\hline Luo R, et al., 2014 & China & Transversal & 848 bebês & 20 pontos/ Nível 4 \\
\hline Jia N, et al., 2015 & China & Transversal & 750 crianças de seis a 36 meses nascidas a termo & 16 pontos/ Nível 4 \\
\hline Dalili H, et al., 2015 & Irã & Transversal & $\mathbf{4 0 0}$ crianças de seis a nove meses & 17 pontos/ Nível 4 \\
\hline Wang F, et al., 2016 & China & Coorte prospectiva & $\mathbf{5 9 . 2 5 0}$ crianças & 18 pontos/ Nível 3 \\
\hline Uyoga MA, et al., 2016 & Quênia & Transversal & 134 crianças de seis a dez meses & 16 pontos/ Nível 4 \\
\hline Clark KM, et al., 2017 & China & Coorte prospectiva & 955 bebês & 18 pontos/ Nível 3 \\
\hline Hong J, et al., 2017 & Coreia & Transversal & 619 bebês & 17 pontos/ Nível 4 \\
\hline Kimura M, et al., 2018 & Japão & Caso-controle & 3.641 bebês com idade de dez meses & 16 pontos/ Nível 3 \\
\hline Wang J, et al., 2020 & China & Caso-controle & 150 lactentes de zero a 24 meses & 16 pontos/ Nível 3 \\
\hline Chen CM, et al., 2020 & China & Transversal & 2.804 bebês saudáveis de um a 12 meses & 18 pontos/ Nível 4 \\
\hline
\end{tabular}

Fonte: Diniz IA, et al., 2022. 
Vinte e três estudos $(82,1 \%)$ avaliaram o aleitamento materno exclusivo e apresentaram média de duração que variaram de inferior a um mês a seis meses (SAARINEN UM, 1978; HOKAMA T, 1993; WANDEL M, et al., 1996; ANTUNES H, et al., 2002; THORSDOTTIR I, et al., 2003; ASSIS AMO, et al., 2004; CHRISTOFIDES A, et al., 2005; CHANTRY CJ, et al., 2007; MONTERROSA EC, et al., 2008; BÜLBÜL I, et al., 2008; CAPOZZI L, et al., 2011; ISOMURA H, et al., 2011; POLAT TB, et al., 2011; LUO R, et al., 2014; DALILI H, et al., 2015; WANG F, et al., 2016; UYOGA MA, et al., 2016; HONG J, et al., 2017; CHEN CM, et al., 2020; KIMURA M, et al., 2018; TANTRACHEEWATHORN S e LOHAJAROENSUB S, 2005; GONG YH, et al., 2008; WANG J, et al., 2020).

Destas vinte e três referências, cinco estudos fizeram comparações do efeito da duração do aleitamento materno exclusivo e anemia por deficiência de ferro por faixas etárias e compararam o nível de hemoglobina com a amamentação exclusiva no quarto e sexto mês (HOKAMA T, 1993; CHRISTOFIDES A, et al., 2005; DALILI H, et al., 2015; HONG J, et al., 2017; KIMURA M, et al., 2018).

Um estudo comparou aos dois, quatro e seis meses (POLAT TB, et al., 2011). Outros autores, Chantry CJ, et al. (2007) compararam em um, quatro e seis meses de vida. Um estudo, Capozzi L, et al. (2011) fez a comparação do nível de hemoglobina aos cinco e seis meses de idade, e outro, Wang F, et al. (2016) comparou nas faixas etárias de menores de três meses, de três a cinco meses, e aos seis meses. Já outros autores avaliaram a associação da duração do aleitamento materno exclusivo com níveis de hemoglobina em um período único. Destas que avaliaram em um único momento, três publicações testaram a associação da anemia ferropriva com o aleitamento materno exclusivo aos quatro meses (TANTRACHEEWATHORN $S$ e LOHAJAROENSUB S, 2005; GONG YH, et al., 2008; WANG J, et al., 2020). Onze estudos avaliaram aos seis meses de idade sem realizar comparações de grupos etários em seus estudos (SAARINEN UM, 1978; ANTUNES H, et al., 2002; THORSDOTTIR I, et al., 2003; ASSIS AMO, et al., 2004; MONTERROSA EC, et al., 2008; WANDEL M, et al., 1996; BÜLBÜL I, et al., 2008; ISOMURA H, et al., 2011; LUO R, et al., 2014; UYOGA MA, et al., 2016; CHEN CM, et al., 2020).

Cinco estudos (17,8\%) avaliaram a amamentação exclusiva prolongada. Estes estudos compararam os níveis de hemoglobina aos seis meses e idade e em idades superiores a seis meses (SITI-NOOR AS, et al., 2006; GONDOLF UH, et al., 2013; CLARK KM, et al., 2017; JIA N, et al., 2015; HOPKINS D, et al., 2007). Nesta faixa etária é considerada como necessário a introdução alimentar complementar (FEWTRELL M, et al., 2017; SBP, 2018).

Em relação ao ponto de corte da hemoglobina para o diagnóstico de anemia, 22 estudos (75\%) consideraram o valor de $<11,0 \mathrm{~g}$ / dl (SAARINEN UM, 1978; HOKAMA T, 1993; WANDEL M, et al., 1996; ANTUNES $\mathrm{H}$, et al., 2002; CHRISTOFIDES A, et al., 2005; TANTRACHEEWATHORN $S$ e LOHAJAROENSUB S, 2005; SITI-NOOR AS, et al., 2006; HOPKINS D, et al., 2007; MONTERROSA EC, et al., 2008; GONG YH, et al., 2008; BÜLBÜL I, et al., 2008; POLAT TB, et al., 2011; LUO R, et al., 2014; JIA N, et al., 2015; DALILI H, et al., 2015; WANG F, et al., 2016; UYOGA MA, et al., 2016; CLARK KM, et al., 2017; HONG J, et al., 2017; WANG J, et al., 2020; KIMURA M, et al., 2018). Este valor é reconhecido pela WHO (2015). Três estudos $(10,7 \%)$ citaram como anemia quando os valores foram inferiores a $10,5 \mathrm{~g} / \mathrm{dl}$ (THORSDOTTIR I, et al., 2003; CHANTRY CJ, et al., 2007; CHEN CM, et al., 2020). Um estudo (3,6\%), Isomura $\mathrm{H}$, et al. (2011), considerou valores inferiores a 10,3 g/dl, outro estudo (3,6\%), Gondolf UH, et al. (2013) refere como anemia o nível de hemoglobina menor que 10,0 g/l, e uma publicação (3,6\%), Assis AMO, et al. (2004) traz como referência valores inferiores a $9 \mathrm{~g} / \mathrm{dl}$. Um estudo $(3,6 \%)$ descreve que avaliou os níveis de acordo com o sexo da criança (CAPOZZI L, et al., 2011) (Quadro 2). 


\section{Hacervo+}

Revista Eletrônica Acervo Saúde | ISSN 2178-2091

Quadro 2 - Variáveis descritivas referente a duração do aleitamento materno exclusivo (AME), nível de hemoglobina e ferritina.

\begin{tabular}{|c|c|c|c|c|}
\hline $\mathbf{N}$ & Referência & Duração do AME & $\begin{array}{l}\text { Ponto de corte do valor da hemoglobina } \\
(\mathrm{Hb}) \text { para considerar anemia }\end{array}$ & $\begin{array}{l}\text { Ponto de corte do valor da } \\
\text { Ferritina para considerar } \\
\text { deficiência de ferro }\end{array}$ \\
\hline 1 & Saarinen U, 1978 & Seis meses & $<11,0 \mathrm{~g} / \mathrm{dL}$ & $<10 \mathrm{~g} / 1$ \\
\hline 2 & Hokama T, 1993 & Quatro meses versus seis meses & $\begin{array}{c}<11,0 \mathrm{~g} / \mathrm{dL} \text { para seis meses de AME/ <9,9 g / } \\
\mathrm{dL} \text { para } 4 \text { meses de AME }\end{array}$ & Não avaliada \\
\hline 3 & Wandel M, et al., 1996 & $\begin{array}{c}\text { Média de 1,5 meses para os turcos e } 3,5 \\
\text { meses para os noruegueses }\end{array}$ & $<110 \mathrm{~g} / \mathrm{L}$ & $<15 \mathrm{ug} / \mathrm{L}$ \\
\hline 4 & Antunes $\mathrm{H}$, et al., 2002 & Média de 4,8 meses & $<110 \mathrm{~g} / \mathrm{L}$ & $<12$ ngll. \\
\hline 5 & Thorsdottir I, et al., 2003 & $\leq$ seis meses & $<10,5 \mathrm{~g} / \mathrm{dl}$. & $<12 \mathrm{mg} / \mathrm{l}$ \\
\hline 6 & Assis AMO, et al., 2004 & $\leq$ seis meses. & $<9 \mathrm{~g} / \mathrm{dl}$ & Não avaliada \\
\hline 7 & Christofides A, et al., 2005 & Quatro a seis meses & $<110 \mathrm{~g} / \mathrm{L}$ & $<8,5 \mathrm{ng} / \mathrm{L}$ \\
\hline 8 & $\begin{array}{l}\text { Tantracheewathorn S } \\
\text { e Lohajaroensub S, } 2005\end{array}$ & Quatro meses & $<11,0 \mathrm{~g} / \mathrm{dl}$ & $<12 \mathrm{ng} / \mathrm{l}$ \\
\hline 9 & Siti-Noor AS, et al., 2006 & $\begin{array}{c}\text { Seis meses versus acima de seis meses } \\
\text { (amamentação prolongada) }\end{array}$ & $<11,0 \mathrm{~g} / \mathrm{dl}$ & $<12 \mathrm{ng} / \mathrm{l}$ \\
\hline 10 & Chantry CJ, et al., 2007 & $\begin{array}{c}\text { Menos de } 1 \text { mês; - De } 1 \text { a } 4 \text { meses; - De } 4 \text { a } \\
6 \text { meses; -Seis meses. }\end{array}$ & $<10,5 \mathrm{~g} / \mathrm{L}$ & $<7 \mu / L$ \\
\hline 11 & Hopkins D, et al., 2007 & Amamentação prolongada aos 8 e 12 meses & $<110 \mathrm{~g} / \mathrm{L}$ & $<16 \mathrm{mg} / \mathrm{l}$ \\
\hline 12 & Monterrosa EC, et al., 2008 & Seis meses & $<110 \mathrm{~g} / \mathrm{L}$ & $<12 \mathrm{mg} / \mathrm{L}$ \\
\hline 13 & Gong YH, et al., 2008 & Do nascimento aos quatro meses & $\leq 110 \mathrm{~g} / \mathrm{L}$ & $<9,85 \mu \mathrm{mol} / \mathrm{L}$ \\
\hline 14 & Bülbül I, et al., 2008 & A duração média de $4,58 \pm 1,80$ meses & $<11 \mathrm{~g} / \mathrm{dL}$ & Não avaliada \\
\hline 15 & Capozzi L, et al., 2011 & Entre $\circ 5^{\circ}$ e $\circ 6^{\circ}$ mês de vida. & $\begin{array}{l}\text { Os valores de } \mathrm{Hb} \text { foram comparados com os } \\
\text { valores percentis esspecíficos do sexo. }\end{array}$ & $<15 \mathrm{ng} / \mathrm{ml}$ \\
\hline 16 & Isomura $\mathrm{H}$, et al., 2011 & Seis meses & $<10,3 \mathrm{~g} / \mathrm{dl}$ & Não avaliada \\
\hline 17 & Polat TB, et al., 2011 & $<2$ meses $/ 2$ a 4 meses $/ ;>4$ meses $/ 6$ meses & $<11 \mathrm{~g} / \mathrm{dL}$ & $<12 \mu g / l$ \\
\hline 18 & Gondolf UH, et al., 2013 & Nove meses & $\leq 100 \mathrm{~g} / \mathrm{l}(6,2 \mathrm{mmol} / \mathrm{I})$ & $\leq 12 \mathrm{mg} / \mathrm{l}$ \\
\hline 19 & Luo R, et al., 2014 & Seis meses & $\leq 110 \mathrm{~g} / \mathrm{L}$ & Não avaliada \\
\hline 20 & Jia N, et al., 2015 & $\begin{array}{l}\text { Aleitamento materno prolongado aos: 6-12 } \\
\text { meses/ } 12-24 \text { meses/ } 24-36 \text { meses }\end{array}$ & $<110 \mathrm{~g} / \mathrm{L}$ & Não avaliada \\
\hline 21 & Dalili H, et al., 2015 & Quatro meses versus seis meses & $<11 \mathrm{mg} / \mathrm{dl})$ & $<12 \mathrm{ng} / \mathrm{dl}$ \\
\hline 22 & Wang F, et al., 2016 & $<3$ meses; $3-5$ meses; 6 meses & $<110 \mathrm{~g} / \mathrm{L}$ & Não avaliada \\
\hline 23 & Uyoga MA, et al., 2016 & Mediana de idade de seis meses. & $<110 \mathrm{~g} / \mathrm{L}$ & $<30 \mu \mathrm{g} / \mathrm{L}$ \\
\hline 24 & Clark KM, et al., 2017 & Nove meses & $<110 \mathrm{~g} / \mathrm{L}$. & $<75 \mu \mathrm{g} / \mathrm{L}$ \\
\hline 25 & Hong J, et al., 2017 & Quatro a seis meses & $<11 \mathrm{~g} / \mathrm{dL}$ & $<12 \mathrm{ng} / \mathrm{mL}$ \\
\hline 26 & Kimura M, et al., 2018 & Quatro a seis meses & $11,0 \mathrm{~g} / \mathrm{dl}$ & Não avaliada \\
\hline 27 & Wang J, et al., 2020 & Quatro meses & $<110 \mathrm{~g} / \mathrm{l}$ & Não avaliada \\
\hline 28 & Chen CM, et al., 2020 & Seis meses & $<10,5 \mathrm{~g} / \mathrm{dL}$ & $<15,0 \mathrm{ng} / \mathrm{mL}$ \\
\hline
\end{tabular}

Fonte: Diniz IA, et al., 2022. 
De um total de 28 publicações, 17 estudos $(60,7 \%)$ apresentaram em seus resultados associação estatística significativa entre a duração do aleitamento materno exclusivo e níveis mais baixos de hemoglobina (HOKAMA T, 1993; CHRISTOFIDES A, et al., 2005; TANTRACHEEWATHORN S e LOHAJAROENSUB S, 2005; SITI-NOOR AS, et al., 2006; CHANTRY CJ, et al., 2007; MONTERROSA EC, et al., 2008; GONG YH, et al., 2008; ISOMURA H, et al., 2011; GONDOLF UH, et al., 2013; LUO R, et al., 2014; DALILI H, et al., 2015; WANG F, et al., 2016; CLARK KM, et al., 2017; HONG J, et al., 2017; WANG J, et al., 2020; CHEN CM, et al., 2020; KIMURA M, et al., 2018).

Deste total três referências avaliaram a associação do aleitamento materno exclusivo após os seis meses de idade (SITI-NOOR AS, et al., 2006; GONDOLF UH, et al., 2013; CLARK KM, et al., 2017).

Seis estudos $(21,4 \%)$ não encontraram associação/correlação da duração do aleitamento materno exclusivo até os seis meses de idade com os níveis de hemoglobina (ANTUNES $H$, et al., 2002; THORSDOTTIR I, et al., 2003; BÜLBÜL I, et al., 2008; CAPOZZI L, et al., 2011; HOPKINS D, et al., 2007; JIA N, et al., 2015).

Embora não apresentem associação com o aleitamento materno exclusivo aos seis meses, duas dessas referências apresentaram associação do aleitamento materno continuado após os seis meses de idade com a deficiência de ferro e anemia ferropriva (HOPKINS D, et al., 2007; JIA N, et al., 2015).

A deficiência de ferro, avaliada a partir dos níveis de ferritina em 19 estudos desta revisão também apresentaram associação com a duração do aleitamento materno exclusivo em 12 publicações (CHRISTOFIDES A, et al., 2005; TANTRACHEEWATHORN S e LOHAJAROENSUB S, 2005; SITI-NOOR AS, et al., 2006; CHANTRY CJ, et al., 2007; HOPKINS D, et al., 2007; MONTERROSA EC, et al., 2008; GONG YH, et al., 2008; DALILI H, et al., 2015; UYOGA MA, et al., 2016; CLARK KM, et al., 2017; HONG J, et al., 2017; CHEN CM, et al., 2020).

Contrapondo os estudos que apresentaram associação da duração do aleitamento materno com a anemia ferropriva, cinco referências $(17,8 \%)$ apontaram associação estatística significativa entre a duração do aleitamento materno exclusivo e níveis altos de hemoglobina comparados a crianças com ausência de aleitamento materno exclusivo (SAARINEN UM, 1978; WANDEL M, et al., 1996; ASSIS AMO, et al., 2004; UYOGA MA, et al., 2016; POLAT TB, et al., 2011)

Conforme preconizado pela WHO (2015) e SBP (2018), treze estudos $(46,4 \%)$ relataram introdução complementar aos seis meses de idade (THORSDOTTIR I, et al., 2003; ASSIS AMO, et al., 2004; CHRISTOFIDES A, et al., 2005; SITI- NOOR AS, et al., 2006; CHANTRY CJ, et al., 2007; HOPKINS D, et al., 2007; MONTERROSA EC, et al., 2008; ISOMURA H, et al., 2011; POLAT TB, et al., 2011; LUO R, et al., 2014; JIA N, et al., 2015; UYOGA MA, et al., 2016; CHEN CM, et al., 2020). Porém nestes estudos essa variável foi pouco explorada e os achados não discutem acerca da qualidade dos alimentos introduzidos na alimentação da criança.

Sete estudos (25\%) revelaram introdução alimentar antes dos seis meses de idade (SAARINEN UM et al., 1978; ANTUNES H, et al., 2002; BÜLBÜL I, et al., 2008; CAPOZZI L, et al., 2011; GONDOLF UH, et al., 2013; HONG J, et al., 2017; TANTRACHEEWATHORN S e LOHAJAROENSUB S, 2005). Oito estudos (28,6\%) não avaliaram a variável referente a introdução complementar de alimentos (HOKAMA T, 1993; WANDEL M, et al., 1996; GONG YH, et al., 2008; DALILI H, et al., 2015; WANG F, et al., 2016; CLARK $\mathrm{KM}$, et al., 2017). Sobre a suplementação profilática de sulfato ferroso, três estudos referem que as crianças foram suplementadas a partir do sexto mês de vida (CAPOZZI L, et al., 2011; UYOGA MA, et al., 2016; CLARK KM, et al., 2017) (Quadro 3). 
Quadro 3 - Nível da hemoglobina e reserva de ferro entre lactentes associado a duração do aleitamento materno exclusivo, suplementação de ferro e introdução alimentar.

\begin{tabular}{|c|c|c|c|c|c|}
\hline $\mathbf{N}$ & Referência & $\begin{array}{l}\text { Suplementação } \\
\text { oral de ferro }\end{array}$ & $\begin{array}{l}\text { Introdução de } \\
\text { alimentos }\end{array}$ & $\begin{array}{l}\text { Associação da duração do aleitamento } \\
\text { materno exclusivo com os níveis de } \\
\text { hemoglobina }\end{array}$ & $\begin{array}{l}\text { Associação da duração do } \\
\text { aleitamento materno exclusivo com } \\
\text { os níveis de ferritina sérica }\end{array}$ \\
\hline 1 & Saarinen U, 1978 & Não avaliou & $\begin{array}{l}\text { Anterior aos seis } \\
\text { meses }\end{array}$ & $\begin{array}{l}\text { Aleitamento materno exclusivo até os seis } \\
\text { meses de idade e nível mais alto de } \\
\text { hemoglobina: associação direta. }\end{array}$ & $\begin{array}{l}\text { Aleitamento materno exclusivo até os } \\
\text { seis meses de idade e níveis baixos de } \\
\text { ferritina sérica: não houve associação } \\
\text { direta. }\end{array}$ \\
\hline 2 & Hokama T, 1993 & Não avaliou & Não avaliou & $\begin{array}{l}\text { Aleitamento materno até os seis meses de } \\
\text { idade e nível mais baixo de hemoglobina: } \\
\text { associação direta. }\end{array}$ & Ferritina não avaliada. \\
\hline 3 & Wandel M, et al., 1996 & Não avaliou & Não avaliou & $\begin{array}{l}\text { Aleitamento materno exclusivo até os seis } \\
\text { meses e estoques mais altos de ferro: } \\
\text { associação direta. }\end{array}$ & $\begin{array}{l}\text { Aleitamento materno exclusivo até os } \\
\text { seis meses de idade e níveis baixos de } \\
\text { ferritina sérica: não houve associação } \\
\text { direta. }\end{array}$ \\
\hline 4 & Antunes H, et al., 2002 & Não avaliou & $\begin{array}{l}\text { Anterior aos seis } \\
\text { meses }\end{array}$ & $\begin{array}{l}\text { Média de aleitamento materno exclusivo } \\
\text { de } 4,8 \text { meses e anemia por deficiência de } \\
\text { ferro: não houve associação direta. }\end{array}$ & $\begin{array}{l}\text { Média de aleitamento materno } \\
\text { exclusivo de } 4,8 \text { meses e níveis baixos } \\
\text { de ferritina sérica: não houve } \\
\text { associação direta. }\end{array}$ \\
\hline 5 & Thorsdottir I, et al., 2003 & Não avaliou & Aos seis meses & $\begin{array}{l}\text { Aleitamento materno exclusivo e anemia } \\
\text { por deficiência de ferro em crianças: não } \\
\text { houve associação direta. }\end{array}$ & $\begin{array}{l}\text { Aleitamento materno exclusivo até os } \\
\text { seis meses de idade e níveis baixos de } \\
\text { ferritina sérica: não houve associação } \\
\text { direta. }\end{array}$ \\
\hline 6 & Assis AMO, et al., 2004 & Não avaliou & Aos seis meses & $\begin{array}{l}\text { Aleitamento materno exclusivo até os seis } \\
\text { meses de idade e níveis altos de } \\
\text { hemoglobina: associação direta; } \\
\text { Aleitamento predominante e níveis altos } \\
\text { de hemoglobina: associação direta. }\end{array}$ & Ferritina não avaliada. \\
\hline 7 & Christofides A, et al., 2005 & Não avaliou & Aos seis meses & $\begin{array}{l}\text { Aleitamento materno exclusivo até os seis } \\
\text { meses de idade e anemia por deficiência } \\
\text { de ferro: associação direta; } \\
\text { Consumo de leite de vaca e anemia por } \\
\text { deficiência de ferro: associacão direta. }\end{array}$ & $\begin{array}{l}\text { Aleitamento materno exclusivo até os } \\
\text { seis meses de idade e níveis baixos de } \\
\text { ferritina sérica: associação direta. }\end{array}$ \\
\hline 8 & $\begin{array}{l}\text { Tantracheewathorn S } \\
\text { e Lohajaroensub S, } 2005\end{array}$ & Não avaliou & $\begin{array}{l}\text { Anterior aos seis } \\
\text { meses }\end{array}$ & $\begin{array}{l}\text { Aleitamento materno até os quatro meses } \\
\text { de idade e anemia por deficiência de ferro: } \\
\text { associação direta. }\end{array}$ & $\begin{array}{l}\text { Aleitamento materno exclusivo até os } \\
\text { quatro meses de idade e níveis baixos } \\
\text { de ferritina sérica: associação direta. }\end{array}$ \\
\hline 9 & Siti-Noor AS, et al., 2006 & Não avaliou & Aos seis meses & $\begin{array}{l}\text { Aleitamento materno exclusivo até os seis } \\
\text { meses e anemia por deficiência de ferro: } \\
\text { associação direta. }\end{array}$ & $\begin{array}{l}\text { Aleitamento materno exclusivo até os } \\
\text { seis meses de idade e níveis baixos de } \\
\text { ferritina sérica: associação direta. }\end{array}$ \\
\hline
\end{tabular}




\begin{tabular}{|c|c|c|c|c|c|}
\hline $\mathbf{N}$ & Referência & $\begin{array}{l}\text { Suplementação } \\
\text { oral de ferro }\end{array}$ & $\begin{array}{l}\text { Introdução de } \\
\text { alimentos }\end{array}$ & $\begin{array}{l}\text { Associação da duração do aleitamento } \\
\text { materno exclusivo com os níveis de } \\
\text { hemoglobina }\end{array}$ & $\begin{array}{l}\text { Associação da duração do } \\
\text { aleitamento materno exclusivo com } \\
\text { os níveis de ferritina sérica }\end{array}$ \\
\hline 10 & Chantry CJ, et al., 2007 & Não avaliou & Aos seis meses & $\begin{array}{l}\text { Aleitamento materno exclusivo até os seis } \\
\text { meses de idade e anemia por deficiência } \\
\text { de ferro: associação direta. }\end{array}$ & $\begin{array}{l}\text { Aleitamento materno exclusivo até os } \\
\text { seis meses de idade e níveis baixos de } \\
\text { ferritina sérica: associação direta. }\end{array}$ \\
\hline 11 & Hopkins D, et al., 2007 & Não avaliou & $\begin{array}{c}\text { Aos seis meses } \\
\text { conferir }\end{array}$ & $\begin{array}{l}\text { Aleitamento materno (> seis mamadas/ } \\
\text { dia) aos oito e doze meses de idade e } \\
\text { níveis baixos de hemoglobina e anemia } \\
\text { por deficiência de ferro: associação direta. }\end{array}$ & $\begin{array}{l}\text { Aleitamento materno exclusivo até os } \\
\text { seis meses de idade e níveis baixos de } \\
\text { ferritina sérica: associação direta. }\end{array}$ \\
\hline 12 & Monterrosa EC, et al., 2008 & Não avaliou & Aos seis meses & $\begin{array}{l}\text { Aleitamento materno exclusivo até os } \\
\text { cinco meses de idade e nível baixo de } \\
\text { hemoglobina: associação direta. }\end{array}$ & $\begin{array}{l}\text { Aleitamento materno exclusivo até os } \\
\text { seis meses de idade e níveis baixos de } \\
\text { ferritina sérica: associação direta. }\end{array}$ \\
\hline 13 & Gong YH, et al., 2008 & Não avaliou & Não avaliou & $\begin{array}{l}\text { Aleitamento materno exclusivo até os } \\
\text { quatro meses, em crianças do sexo } \\
\text { masculino e anemia por deficiência de } \\
\text { ferro: associação direta }\end{array}$ & $\begin{array}{l}\text { Aleitamento materno exclusivo até os } \\
\text { quatro meses de idade e níveis baixos } \\
\text { de ferritina sérica: associação direta. }\end{array}$ \\
\hline 14 & Bülbül I, et al., 2008 & Não avaliou & $\begin{array}{c}\text { Anterior aos seis } \\
\text { meses }\end{array}$ & $\begin{array}{l}\text { Aleitamento materno exclusivo e nível de } \\
\text { hemoglobina: não houve correlação } \\
\text { estatisticamente significativa. }\end{array}$ & Ferritina não avaliada. \\
\hline 15 & Capozzi L, et al., 2011 & Sim & $\begin{array}{c}\text { Anterior aos seis } \\
\text { meses }\end{array}$ & $\begin{array}{l}\text { Aleitamento materno exclusivo e anemia } \\
\text { por deficiência de ferro: não houve } \\
\text { correlação estatisticamente significativa. }\end{array}$ & $\begin{array}{l}\text { Aleitamento materno exclusivo até os } \\
\text { seis meses de idade e níveis baixos de } \\
\text { ferritina sérica: não houve associação } \\
\text { direta. }\end{array}$ \\
\hline 16 & Isomura H, et al., 2011 & Não avaliou & Aos seis meses & $\begin{array}{l}\text { Aleitamento materno exclusivo até os seis } \\
\text { meses de idade e nível baixo de } \\
\text { hemoglobina: associação direta }\end{array}$ & Ferritina não avaliada. \\
\hline 17 & Polat TB, et al., 2011 & Não avaliou & Aos seis meses & $\begin{array}{l}\text { Aleitamento materno exclusivo até os seis } \\
\text { meses de idade e nível mais alto de } \\
\text { hemoglobina: associação direta }\end{array}$ & $\begin{array}{l}\text { Aleitamento materno exclusivo até os } \\
\text { seis meses de idade e níveis baixos de } \\
\text { ferritina sérica: não houve associação } \\
\text { direta. }\end{array}$ \\
\hline 18 & Gondolf UH, et al., 2013 & Não avaliou & $\begin{array}{c}\text { Anterior aos seis } \\
\text { meses }\end{array}$ & $\begin{array}{l}\text { Duração do aleitamento materno } \\
\text { exclusivo após seis meses e anemia por } \\
\text { deficiência ferro: associação direta. }\end{array}$ & $\begin{array}{l}\text { Aleitamento materno exclusivo até os } \\
\text { seis meses de idade e níveis baixos de } \\
\text { ferritina sérica: não houve associação } \\
\text { direta. }\end{array}$ \\
\hline 19 & Luo R, et al., 2014 & Não avaliou & Aos seis meses & $\begin{array}{l}\text { Amamentação exclusiva ou } \\
\text { predominantemente até seis meses de } \\
\text { idade, bebês amamentados por mais de } \\
\text { seis meses de idade e níveis baixos de } \\
\text { hemoglobina: associação direta. }\end{array}$ & Ferritina não avaliada. \\
\hline
\end{tabular}




\begin{tabular}{|c|c|c|c|c|c|}
\hline $\mathbf{N}$ & Referência & $\begin{array}{l}\text { Suplementação } \\
\text { oral de ferro }\end{array}$ & $\begin{array}{l}\text { Introdução de } \\
\text { alimentos }\end{array}$ & $\begin{array}{l}\text { Associação da duração do aleitamento } \\
\text { materno exclusivo com os níveis de } \\
\text { hemoglobina }\end{array}$ & $\begin{array}{l}\text { Associação da duração do } \\
\text { aleitamento materno exclusivo com } \\
\text { os níveis de ferritina sérica }\end{array}$ \\
\hline 20 & Jia N, et al., 2015 & Não avaliou & Aos seis meses & $\begin{array}{l}\text { Aleitamento materno exclusivo até seis } \\
\text { meses de idade e a anemia por } \\
\text { deficiência de ferro: não houve } \\
\text { associação direta- Duração do } \\
\text { aleitamento materno exclusivo após seis } \\
\text { meses e anemia por deficiência ferro: } \\
\text { associação direta }\end{array}$ & Ferritina não avaliada. \\
\hline 21 & Dalili H, et al., 2015 & Não avaliou & Não avaliou & $\begin{array}{l}\text { Aleitamento materno exclusivo até os seis } \\
\text { meses de idade, aleitamento materno } \\
\text { prolongado e anemia por deficiência de } \\
\text { ferro: associação direta }\end{array}$ & $\begin{array}{l}\text { Aleitamento materno exclusivo até os } \\
\text { seis meses de idade e níveis baixos de } \\
\text { ferritina sérica: associação direta. }\end{array}$ \\
\hline 22 & Wang F, et al., 2016 & Não avaliou & Não avaliou & $\begin{array}{l}\text { Aleitamento materno exclusivo até os seis } \\
\text { meses de idade e anemia por deficiência } \\
\text { de ferro: associação direta. }\end{array}$ & Ferritina não avaliada. \\
\hline 23 & Uyoga MA, et a.I, 2016 & Sim & Aos seis meses & $\begin{array}{l}\text { Aleitamento materno exclusivo até os seis } \\
\text { meses de idade e níveis mais altos de } \\
\text { hemoglobina: associação direta. }\end{array}$ & $\begin{array}{l}\text { Aleitamento materno exclusivo até os } \\
\text { seis meses de idade e níveis baixos de } \\
\text { ferritina sérica: associação direta. }\end{array}$ \\
\hline 24 & Clark KM, et al., 2017 & Sim & Não avaliou & $\begin{array}{l}\text { O aleitamento materno exclusivo até os } \\
\text { seis meses de idade e anemia por } \\
\text { deficiência de ferro: associação direta } \\
\text { Duração do aleitamento materno } \\
\text { exclusivo após seis meses e anemia por } \\
\text { deficiência ferro: associação direta }\end{array}$ & $\begin{array}{l}\text { Aleitamento materno exclusivo até os } \\
\text { seis meses de idade e níveis baixos de } \\
\text { ferritina sérica: associação direta. }\end{array}$ \\
\hline 25 & Hong J, et al., 2017 & Não avaliou & $\begin{array}{l}\text { Anterior seis } \\
\text { meses }\end{array}$ & $\begin{array}{l}\text { Aleitamento materno exclusivo até os seis } \\
\text { meses de idade e anemia por deficiência } \\
\text { de ferro: associação direta }\end{array}$ & $\begin{array}{l}\text { Aleitamento materno exclusivo até os } \\
\text { seis meses de idade e níveis baixos de } \\
\text { ferritina sérica: associação direta. }\end{array}$ \\
\hline 26 & Kimura M, et al., 2018 & Não avaliou & Não avaliou & $\begin{array}{l}\text { Aleitamento materno exclusivo até seis } \\
\text { meses de idade e anemia por deficiência } \\
\text { de ferro: associação direta. }\end{array}$ & Ferritina não avaliada. \\
\hline 27 & Wang J, et al., 2020 & Não avaliou & Não avaliou & $\begin{array}{l}\text { Aleitamento materno exclusivo até os } \\
\text { quatro meses de idade e anemia por } \\
\text { deficiência de ferro: associação direta }\end{array}$ & Ferritina não avaliada. \\
\hline 28 & Chen CM, et al., 2020 & Não avaliou & Aos seis meses & $\begin{array}{l}\text { Aleitamento materno exclusivo até seis } \\
\text { meses de idade e anemia por deficiência } \\
\text { de ferro: associação direta }\end{array}$ & $\begin{array}{l}\text { Aleitamento materno exclusivo até os } \\
\text { seis meses de idade e níveis baixos de } \\
\text { ferritina sérica: associação direta. }\end{array}$ \\
\hline
\end{tabular}

Fonte: Diniz IA, et al., 2021.

REAS | Vol.15(2) | DOI: https://doi.org/10.25248/REAS.e9610.2022 $\quad$ Página 13 de 19




\section{DISCUSSÃO}

Os principais achados dessa revisão evideciaram que a maioria dos estudos analisados apontaram uma associação estatisticamente significativa entre a duração do aleitamento materno exclusivo até os seis meses de idade e níveis baixos de hemoglobina. Também foi demonstrado entre os estudos que avaliaram a ferritina sérica, a associação da duração do aleitamento materno exclusivo aos seis meses e a deficiência de ferro.

$\mathrm{Na}$ atualização desta revisão identificou-se dois estudos que também se referem a associaçao do aleitamento materno exclusivo até aos seis meses com o risco de anemia por deficiência de ferro. Estes estudos sugerem que $o$ aleitamento materno exclusivo mantido entre quatro e seis meses aumentam o risco de anemia por deficiência de ferro (SUWANNAKEEREE P e JANGMEONWAI P, 2020; PURANITEE PMD, et al., 2021).

Contudo estes dados devem ser analisados com cautela, considerando que variáveis como a idade da criança, momento da introdução alimentar, a qualidade dos alimentos oferecidos, o não uso de alimentos fortificados com ferro, bem como intercorrências ocasionais de infecções das vias respiratórias e diarreias, e a não suplementação profilática de sulfato ferroso em tempo oportuno, podem interferir no desfecho da anemia por deficiência de ferro (ANDRE HP, et al., 2018, SILVA LLS, et al., 2018; AMARAL SM, et al., 2021; SBP, 2018). Portanto, esses fatores significativos precisariam ser melhor descritos para que a duração do aleitamento materno exclusivo pudesse ser de fato avaliado em sua relação ou não com a ocorrência de anemia por deficiência de ferro. Assim, reforçando a importância de investigação no que tange a duração do aleitamento materno exclusivo e anemia por deficiência de ferro em lactentes.

No Brasil esta situação tem interferido em ações governamentais e não governamentais no intuito de controlar a anemia nesta população específica. Apesar da adoção de medidas profiláticas, educativas e de alterações no ambiente, o índice da anemia continua a apresentar valores consideravelmente altos (SBP, 2018; CASTRO IRR, et al., 2021).

A alta prevalência de anemia por deficiência de ferro e aparente ineficácia de seu controle tem sido descrita na literatura. Pesquisa seccional realizada em 2021, com amostra probabilística de crianças, entre a faixa etária de seis a 59 meses de idade, verificou uma alta prevalência de anemia por deficiência de ferro em crianças com menos de 24 meses. Contudo, o crescimento da prática de aleitamento materno exclusivo até os seis meses de idade e de aleitamento materno entre crianças menores de 24 meses, providências adotadas pelos órgãos governamentais de saúde como a fortificação de farinhas não parecem esclarecer 0 resultado encontrado (CASTRO IRR, et al., 2021).

Compreende-se que a alimentação tem o papel primordial na prevenção da anemia por deficiência de ferro e na melhora do estado nutricional. No entanto, o consumo alimentar em crianças menores de 24 meses ainda está distante do aconselhado, há evidências de introdução precoce de alimentos pobres em ferro, especialmente em idades anteriores aos seis meses de idade (AMARAL SM, et al., 2021).

Uma investigação seccional, realizada em quatro cidades brasileiras, Rio Branco, Olinda, Goiânia e Porto Alegre, com 520 crianças, entre a faixa etária de 11 a 15 meses de idade, indicou uma prevalência de $23,1 \%$ de anemia por deficiência de ferro em crianças que receberam a alimentação complementar inadequada (SILVA LLS, et al., 2018). Porto JP, et al. (2018), em uma coorte prospectiva, com 286 crianças acompanhadas do nascimento aos doze meses de idade no município de Vitória da Conquista - Bahia confirmaram que as frequências de introdução de quatro ou mais alimentos ultraprocessados e, de aleitamento materno exclusivo por menos de 120 dias foram elevadas, constituindo fator de risco para surgimento de doenças como por exemplo anemia por deficiência de ferro.

É consenso na literatura que a introdução alimentar da criança quando iniciada anterior a idade de seis meses, inclusive com a ingestão de carne, aumenta o risco de anemia nas crianças (SALAMI A, et al., 2018; ANDRÉ HP, et al., 2018). Ao contrário, a alimentação complementar tardia, após 240 dias, também tem sido um determinante de anemia em lactente (OLIVEIRA CS, et al., 2016). 
Neste sentido a amamentação exclusiva prolongada discutida por alguns autores, Siti-Nor AS, et al. (2006); Clark KM, et al. (2017) e Gondolf UH, et al. (2013) comprometem a avaliação do efeito do leite materno sobre o evento deficiência de ferro e anemia por deficiência de ferro, considerando que o contexto nutricional como um todo, e não somente a amamentação, interfere neste processo, pois compreende-se que a amamentação após os seis meses de vida precisa estar associada as práticas alimentares adequadas.

Alguns estudos fazem comparações dos tipos de aleitamento e sua relação com os níveis de hemoglobina. Estes estudos não evidenciam diferenças significativas nos níveis de hemoglobina entre o leite materno e o uso de fórmula (SAARINEN UM, 1978; HOKAMA T, 1993; WANDEL M, et al., 1996; CHRISTOFIDES A, et al., 2005; TANTRACHEEWATHORN S e LOHAJAROENSUB S , 2005; MONTERROSA EC, et al., 2008; GONG YH, et al., 2008; LUO R, et al., 2014; HONG J, et al., 2017; CHEN CM, et al., 2020) .

Contudo, em relação ao leite de vaca, os níveis da hemoglobina tendem a ser menores. O leite de vaca não é recomendado antes dos 24 meses de idade (FEWTRELL M, et al., 2017; SBP, 2018). Após os quatro meses de vida estima-se uma diminuição de $0,2 \mathrm{~g} / \mathrm{dl}$ nos níveis de hemoglobina a cada mês de uso do leite de vaca (SBP, 2018). Portanto, considera-se que este de tipo de aleitamento interfere no aumento da anemia ferropriva em crianças (VICTORA CG, et al., 2016; WHO, 2017; SBP, 2018). Os estudos que fazem análise do leite de vaca e anemia ferropriva nesta revisão corroboram a literatura vigente.

Ao comparar o leite de origem animal com a amamentação, embora o leite materno revele uma baixa quantidade de ferro $(0,5-1,0 \mathrm{mg}$ de ferro/litro), sua biodisponibilidade é considerada de excelência (cerca de $50 \%$ do ferro é absorvido), sendo suficiente para assegurar um ótimo balanço de ferro em crianças nascidas a termo, até os seis meses de vida (SBP, 2018). Contudo, a ingestão precoce de alimentos complementares pode reduzir a biodisponibilidade do ferro em até $80 \%$, consequentemente favorecendo o aparecimento da anemia ferropriva no lactente (SILVA LLS, et al., 2018).

A despeito da suplementação profilática com sulfato ferroso a maioria dos estudos não avalia o uso (SAARINEN UM, 1978; HOKAMA T, 1993; WANDEL $M$, et al., 1996; ANTUNES $\mathrm{H}$, et al., 2002; THORSDOTTIR I, et al., 2003; ASSIS AMO, et al., 2004; CHRISTOFIDES A, et al., 2005; TANTRACHEEWATHORN S e LOHAJAROENSUB S, 2005; CHANTRY CJ, et al., 2007; HOPKINS D. et al., 2007; MONTERROSA EC, et al., 2008; GONG YH, et al., 2008; BÜLBÜL I, et al., 2008; ISOMURA H, et al., 2011; POLAT TB, et al., 2011; GONDOLF UH, et al., 2013; LUO R, et al., 2014; JIA N, et al., 2015; DALILI H, et al., 2015, WANG F, et al., 2016, HONG J, et al., 2017; WANG J, et al., 2020).

Esta é uma variável importante e pode exercer influência no desfecho da anemia por deficiência de ferro. Somente três estudos descrevem sobre a prática da suplementação profilática do ferro, e nestes estudos observou-se que essa conduta foi realizada após os seis meses de idade da criança (CAPOZZI L, et al., 2011; UYOGA MA, et al., 2016; CLARK KM, et al., 2017). Isto pode ter favorecido a ocorrência de anemia por deficiência de ferro.

A Sociedade Brasileira de Pediatria recomenda a suplementação profilática dos seis aos 24 meses de idade, e nos lactentes que apresentam condições de risco provinientes da gestação e nascimento essa introdução deve ocorrer a partir de três meses (SBP, 2021). Uma investigação seccional, em Divinópolis, Brasil, com 65 crianças, entre a faixa etária de seis e 18 meses de idade, verificou que $70,5 \%$ das mães tinham dificuldade em administrar o suplemento de sulfato ferroso à criança, sendo o esquecimento o principal motivo (GONTIJO TL, et al., 2017). Em 2018, um estudo exploratório com análise qualitativa dos dados, desenvolvido em um município do interior do estado de São Paulo com 12 mães de crianças entre seis meses e 11 meses e 29 dias de idade, constatou um raso conhecimento das mães acerca da anemia por deficiência de ferro, sua definição capacidade de admitir a precisão do uso do sulfato ferroso como medida preventiva (HENRIQUE NCP, et al., 2018).

Corroborando com esses achados, Cembranel F, et al. (2017), em um estudo transversal, utilizando dados secundários de 13.197 crianças menores de dois anos de idade atendidos nos centros de saúde da rede pública de Florianópolis, Brasil, identificou que somente 2,4\% iniciaram e incluíram a suplementação com sulfato ferroso dentro da idade e periocidade recomendada. 
Contraponpondo os estudos que apresentaram associação da duração do aleitamento materno exclusivo com deficiência de ferro e/ou anemia por deficiência de ferro, cinco estudos apresentaram resultados em que a duração do aleitamento materno exclusivo até os seis meses de idade elevou o nível da hemoglobina das crianças avaliadas (SAARINEN UM, 1978; WANDEL M, et al., 1996; ASSIS AMO, et al., 2004; UYOGA MA, et al., 2016; POLAT TB, et al., 2011). Um dos estudos identificados na atualização desta revisão, também descreve que ser amamentado exclusivamente até os seis meses reduziu consideravelmente a probabilidade de anemia por deficiência de ferro, o que coloca o aleitamento materno exclusivo como fator protetivo para a ocorrência de anemia (TAKELE WW, et al., 2021).

Estes achados vão de encontro com alguns manuscritos publicados na literatura que apontam associação entre aleitamento materno exclusivo por mais tempo a um melhor status de ferro nas crianças de até 24 meses de idade (WHO, 2017; PÉREZ-ESCAMILLA R, et al., 2019).

Um aspecto importante é em relação ao gênero da criança, que parece exercer influência nos níveis da hemoglobina. Duas publicações dessa revisão, Gong YH, et al. (2008) e Kimura M, et al. (2018) apresentaram resultados de ocorrência de anemia por deficiência de ferro e duração do aleitamento materno exclusivo entre as crianças do sexo masculino. Esse achado se consolida com a literatura. A anemia ferropriva ocorre por motivos variados, mas ao que se refere à criança, o peso ao nascer, sexo masculino, desnutrição, presença de infecção, deficiência de outros micronutrientes e idade inferior a 24 meses, têm sido descritos como fatores associados à anemia ferropriva (ANDRE HP, et al., 2018).

Outra vertente interessante averiguada nesta revisão sistemática foi pertinente ao país que mais investigou sobre a duração do aleitamento materno e anemia por deficiência de ferro, a China. A Associação de Nutrição da China sugere que as mães amamentem exclusivamente seus bebês desde o nascimento até os quatro meses e, após esse período amamentem seus filhos com leites complementares. De acordo com as tradições chinesas, as mães que amamentam seus bebês têm amplo respeito e apoio da família. Porém, a prática do aleitamento materno na China têm sido cada vez menos preferida (HOKAMA T, 1993).

Ademais, com desenvolvimento da China, há competições de recursos humanos. Assim, a amamentação exclusiva até os seis meses pode afetar a vida profissional das mães. Corroborando com esse panorama, uma investigação seccional, feita em cinco cidades típicas da China: Xangai, Nanquim, Pequim, Shenzhen, Chengdu, com 750 crianças, mostrou uma prevalência de 17,2\% de anemia entre lactentes de seis a 24 meses, pertencentes à área urbana (JIA N, et al., 2015).

Enfim, esta revisão apresenta limitações para responder se a duração do aleitamento materno exclusivo até os seis meses pode influenciar na ocorrência de deficiênca de ferro e anemia por deficiência de ferro. Isto porque outras variáveis confundidoras precisariam ser melhor exploradas em estudos para que a duração do aleitamento materno exclusivo pudesse de fato ser avaliada em sua relação ou não com a ocorrência de anemia por deficiência de ferro. Por outro lado, essa revisão se fez importante por levantar esta lacuna presente na literatura.

\section{CONSIDERAÇÕES FINAIS}

Neste sentido, compreende-se que a associação da duração do aleitamento materno exclusivo com a anemia por deficiência de ferro carece ser melhor investigada em estudos longitudinais que controle as variáveis envolvidas no desfecho da anemia por deficiência de ferro em lactentes, como a história de anemia materna na gestação e puerpério, alimentação complementar, profilaxia de sulfato ferroso e clampeamento de cordão umbilical. Cabe aqui ressaltar que o leite materno é mais do que alimento, e diante de todos os seus benefícios, dentre eles os relacionados aos fatores imunológicos, de desenvolvimento infantil, metabólico e psicossomático, é que se reforça a orientação de que o leite materno deve ser mantido exclusivamente até os seis meses de idade e até aos 24 meses de forma complementar, conforme preconizado pela Organização Mundial da Saúde.

Registro no prospero: CRD42020186422. 


\section{REFERÊNCIAS}

1. AMARAL SM, et al. Anemia ferropriva na infância: causas e consequências. Revista de Casos e Consultoria, 2021; 12(1): e23991-e23991.

2. ANDRE HP, et al. Indicadores de insegurança alimentar e nutricional associados à anemia ferropriva em crianças brasileiras: uma revisão sistemática. Ciênc. saúde coletiva, 2018; 23(4): 1159-1167.

3. ANTUNES $\mathrm{H}$, et al. Prevalência de anemia por deficiência de ferro de acordo com o tipo de alimentação do lactente. Acta Médica Portuguesa, 2002; 15: 193-197.

4. ASSIS AMO, et al. Hemoglobin concentration, breastfeeding and complementary feeding in the first year of life. Revista de saude publica, 2004; 38: 543-551.

5. BALDONI NR, et al. Prevalence of overweight and obesity in the adult indigenous population in Brazil: a systematic review with meta-analysis. Diabetes \& Metabolic Syndrome: Clinical Research \& Reviews, 2019; 13(3): 1705-1715.

6. BÜLBÜL $L$, et al. Correlation between exclusive breast feeding duration and blood hemoglobin levels, erythrocyte indices in six-month-old infants. Turk Arch Pediatr., 2008; 43: 119-126.

7. CAI C, et al. Effect of Daily Iron Supplementation in Healthy Exclusively Breastfed Infants: A Systematic Review with Meta-Analysis. Breastfeeding medicine: the official journal of the Academy of Breastfeeding Medicine, 2017; 12(10): 597-603.

8. CAPOZZI L, et al. Effect on haematological and anthropometric parameters of iron supplementation in the first 2 years of life. Risks and benefits. Hematology (Amsterdam, Netherlands), 2011; 16(5): 261-264.

9. CASTRO IRR, et al. Prevalência de anemia e deficiência de vitamina A e consumo de ferro e de vitamina A entre crianças usuárias do Sistema Único de Saúde na cidade do Rio de Janeiro, Brasil. Cad. Saúde Pública, 2021; 37(4): e00252420.

10. CEMBRANEL $F$, et al. Inadequações no tratamento da anemia ferropriva entre crianças cadastradas no programa nacional de suplementação de ferro em Florianópolis, Santa Catarina. Texto \& ContextoEnfermagem, 2017; 26(2): e06310015.

11. CHANTRY CJ, et al. Full breastfeeding duration and risk for iron deficiency in US infants. Breastfeeding Medicine, 2007; 2(2): 63-73.

12. CHEN CM, et al. Iron Status of Infants in the First Year of Life in Northern Taiwan. Nutrients, 2020; 12(1): 139.

13. CHRISTOFIDES A, et al. Iron deficiency and anemia prevalence and associated etiologic risk factors in First Nations and Inuit communities in Northern Ontario and Nunavut. Canadian journal of public health = Revue canadienne de sante publique, 2005; 96(4): 304-307

14. CLARK KM, et al. Breastfeeding, Mixed, or Formula Feeding at 9 Months of Age and the Prevalence of Iron Deficiency and Iron Deficiency Anemia in Two Cohorts of Infants in China. The Journal of pediatrics, 2017; 181: 56-61.

15. DALILI H, et al. Is there any relation between Duration of breastfeeding and anemia?. Iranian journal of pediatric hematology and oncology, 2015; 5(4): 218-226.

16. DOMELLÖF M, et al. Iron requirements of infants and toddlers. Journal of pediatric gastroenterology and nutrition, 2014; 58(1): 119-129.

17. DOWNS SH, BLACK N. The feasibility of creating a checklist for the assessment of the methodological quality both of randomised and non-randomised studies of health care interventions. Journal of epidemiology and community health, 1998; 52(6): 377-384.

18. FEWTRELL M, et al. Complementary feeding: a position paper by the European Society for Paediatric Gastroenterology, Hepatology, and Nutrition (ESPGHAN) Committee on Nutrition. Journal of pediatric gastroenterology and nutrition, 2017; 64(1): 119-132.

19. FISBERG M, et al. Consenso sobre anemia ferropriva: mais que umadoença, uma urgência médica! Departamentos de Nutrologia e Hematologia-Hemoterapia. Sociedade Brasileira de Pediatria, 2018; 2.

20. GALVÃO CM. Níveis de Evidência. Acta Paul Enferm., 2006; 19(2): 5.

21. GONDOLF UH, et al. Iron supplementation is positively associated with increased serum ferritin levels in 9-month-old Danish infants. The British journal of nutrition, 2013; 109(1): 103-110.

22. GONG YH, et al. Correlation of 4-month infant feeding modes with their growth and iron status in Beijing. Chinese medical journal, 2008; 121(5): 392-398. 
23. GONTIJO TL, et al. Prática profilática da anemia ferropriva em crianças na estratégia saúde da família. Revista de Enfermagem do Centro-Oeste Mineiro, 2017; 19(2): 293-307.

24. HENRIQUE NCP, et al. Anemia ferropriva e o uso do sulfato ferroso: facilidades e dificuldades na prevenção. Revista Enfermagem UERJ, 2018; 26: 37232.

25. HOKAMA T. A study of the hemoglobin levels in breast-fed infants in one village of Okinawa prefecture. Pediatrics International., 1993; 35(2): 138-140.

26. HONG J, et al. Breastfeeding and Red Meat Intake Are Associated with Iron Status in Healthy Korean Weaning-age Infants. Journal of Korean medical science, 2017; 32(6): 974-984.

27. HOPKINS D, et al. Infant feeding in the second 6 months of life related to iron status: an observational study. Archives of disease in childhood, 2007; 92(10): 850-854.

28. INDICATORS IQ. Agency for Healthcare Research and Quality, 2002. Disponível em: https://qualityindicators.ahrq.gov/. Acessado em: 20 de dezembro de 2021.

29. ISOMURA $H$, et al. Type of milk feeding affects hematological parameters and serum lipid profile in Japanese infants. Pediatrics international: official journal of the Japan Pediatric Society, 2011; 53(6): 807813.

30. JIA N, et al. Dietary survey of anaemic infants and young children in urban areas of China: a cross-sectional study. Asia Pacific journal of clinical nutrition, 2015; 24(4): 659-664.

31. KIMURA M, et al. High prevalence of anemia in 10-month-old breast-fed Japanese infants. Pediatrics international: official journal of the Japan Pediatric Society, 2018; 60(7): 651-655.

32. LUO R, et al. Anemia and feeding practices among infants in rural Shaanxi Province in China. Nutrients, 2014; 6(12): 5975-5991.

33. MONTERROSA EC, et al. Predominant breast-feeding from birth to six months is associated with fewer gastrointestinal infections and increased risk for iron deficiency among infants. The Journal of nutrition, 2008; 138(8): 1499-1504.

34. NOGUEIRA-DE-ALMEIDA CA, et al. Prevalence of childhood anaemia in Brazil: still a serious health problem: a systematic review and meta-analysis. Public health nutrition, 2021; 24(18): 6450-6465.

35. OLIVEIRA CS, et al. Anemia and micronutrient deficiencies in infants attending at Primary Health Care in Rio Branco, Acre, Brazil. Ciência \& Saúde coletiva, 2016; 21(2): 517-529.

36. OUZZANI M, et al. Rayyan - a web and mobile app for systematic reviews. System rev, 2016; 5(1): 1-10.

37. PAGE MJ, et al. The PRISMA 2020 statement: an updated guideline for reporting systematic reviews. Bmj, $2021 ; 372$.

38. PÉREZ-ESCAMILLA R, et al. Perspective: Should Exclusive Breastfeeding Still Be Recommended for 6 Months? Advances in Nutrition, 2019; 10(6): 931-943.

39. POLAT TB, et al. Evaluation of Iron Status in Relation to Feeding Practices in Early Infancy. Macedonian Journal of Medical Sciences, 2011; 4(1): 70-74.

40. PORTO JP, et al. Aleitamento materno exclusivo e introdução de alimentos ultraprocessados no primeiro ano de vida: estudo de coorte no sudoeste da Bahia. Epidemiol. Serv. Saúde, 2018; 30(2).

41. PURANITEE PMD, et al. Determination of Hemoglobin Level Among 9-Month-Old Infants Visiting Well Child Clinic. Global Pediatric Health, 2021, 8: 1-7.

42. SAARINEN UM. Need for iron supplementation in infants on prolonged breast feeding. The Journal of pediatrics, 1998; 93(2): 177-180.

43. SALAMI A, et al. Prevalence of anemia among Lebanese hospitalized children: Risk and protective factors. PLoS One, 2018; 13(8): e0201806.

44. SILVA LLS, et al. Factors associated with anemia in young children in Brazil. PLoS One, 2018; 25(1): 1 -12

45. SITI-NOOR AS, et al. Prevalence and risk factors for iron deficiency in Kelantanese pre-school children. Singapore medical journal, 2006; 47(11): 935.

46. SOCIEDADE BRASILEIRA DE PEDIATRIA. Manual de orientação para a alimentação do lactente, do préescolar, do escolar, do adolescente e na escola, 2018. Disponível em: https://www.sbp.com.br/fileadmin/user_upload/pdfs/14617a-PDManualNutrologia-Alimentacao.pdf. Acessado em: 20 de dezembro de 2021.

47. SOCIEDADE BRASILEIRA DE PEDIATRIA. Consenso sobre Anemia ferropriva - Atualização: destaques 2021. Disponível em: https://www.sbp.com.br/fileadmin/user_upload/23172c-Diretrizes. Acessado em: 20 de dezembro de 2021. 
48. SUWANNAKEEREE P, JANGMEONWAI P. The Prevalence and Risk Factors of Iron Deficiency Anemia in Thai Infants by Complete Blood Count at 9-Month-Old. J Med Assoc Thai, 2020; 103(9): 891-6.

49. TAKELE WW, et al. Anemia and Contributing Factors in Severely Malnourished Infants and Children Aged between 0 and 59 Months Admitted to the Treatment Centers of the Amhara Region, Ethiopia: A Multicenter Chart Review Study. Hindawi, 2021: 6636043.

50. TANTRACHEEWATHORN S, \& LOHAJAROENSUB S. Incidence and risk factors of iron deficiency anemia in term infants. J Med Assoc Thai, 2005; 88(1): 45-51.

51. THORSDOTTIR I, et al. Iron status at 12 months of age -- effects of body size, growth and diet in a population with high birth weight. European journal of clinical nutrition, 2003; 57(4): 505-513.

52. UYOGA MA, Duration of exclusive breastfeeding is a positive predictor of iron status in 6-to 10-month-old infants in rural Kenya. Maternal \& child nutrition, 2017; 13(4): e12386.

53. VICTORA CG, et al. Breastfeeding in the 21st century: epidemiology, mechanisms, and lifelong effect. The Lancet, 2016; 387(10017): 475-490.

54. WANDEL M, et al. Iron status and weaning practices among Norwegian and immigrant infants. Nutrition Research, 1996; 16(2): 251-265.

55. WANG F, et al. Prolonged exclusive breastfeeding duration is positively associated with risk of anemia in infants aged 12 months. The Journal of nutrition, 2016; 146(9): 1707-1713.

56. WANG J, et al. A Retrospective Case-Control Study of the Determinants of Iron Deficiency Anemia in Infants in an Urban Community in Shanghai, China Between 2010-2015. Medical science monitor: international medical journal of experimental and clinical research, 2020; 26: e921463.

57. WORLD HEALTH ORGANIZATION. The global prevalence of anaemia in 2011. 2015. Disponível em: https://apps.who.int/iris/bitstream/handle/10665/177094/9789241564960_eng.pdf. Acessado em: 20 de dezembro de 2021.

58. WORLD HEALTH ORGANIZATION. Nutritional anaemias: tools for effective prevention and control. 2017. Disponível em: https://www.who.int/publications/i/item/9789241513067. Acessado em: 20 de dezembro de 2021. 